\title{
CXCR4 is involved in CD133-induced EMT in non-small cell lung cancer
}

\author{
ZHENBO TU $^{1 *}$, SONGPING XIE ${ }^{2 *}$, MENG XIONG $^{1 *}$, YINCHU LIU $^{3}$, XIANGYONG YANG ${ }^{4}$, \\ KINGSLEY MIYANDA TEMBO ${ }^{1}$, JIE HUANG ${ }^{2}$, WEIDONG HU ${ }^{5}$, XIAOXING HUANG ${ }^{1}$, SHAN PAN $^{1}$,

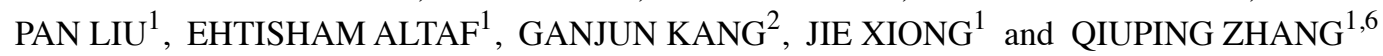 \\ ${ }^{1}$ Department of Immunology, School of Basic Medical Sciences, Wuhan University; ${ }^{2}$ Department of Thoracic Surgery, \\ Renmin Hospital of Wuhan University; ${ }^{3}$ School of Basic Medical Sciences, Wuhan University; \\ ${ }^{4}$ Engineering and Technology College, Hubei University of Technology; ${ }^{5}$ Department of Thoracic Oncology, \\ Zhongnan Hospital and ${ }^{6}$ Hubei Provincial Key Laboratory of Developmentally Originated Disease, \\ School of Basic Medical Sciences, Wuhan University, Wuhan, Hubei, P.R. China
}

Received September 2, 2016; Accepted November 3, 2016

DOI: 10.3892/ijo.2016.3812

\begin{abstract}
Metastasis is the major cause of death in patients with non-small cell lung cancer (NSCLC), and epithelialmesenchymal transition (EMT) has been observed to be one of the key regulators of metastasis in certain cancers as it confers an invasive phenotype. CD133 is a widely used cancer stem cell (CSC) marker, and CD133-positive cancer cells are thought to be tumor-initiating cells with CSC characteristics, while CXCR4, a stromal-derived-factor-1 specific chemokine receptor, is highly expressed in NSCLC tissues and participates in cancer progression by regulating cell anti-apoptosis. We previously demonstrated that CXCR4 promotes NSCLC chemoresistance by upregulating CYP1B1, however, the relationship of CD133, CXCR4 and EMT processes in NSCLC metastasis are unclear. In this study, we detected a CD133 and CXCR4 high expression in tissue specimens from 64 NSCLC patients by immunohistochemistry, of which CD133 and CXCR4 were found to be positively associated with metastatic NSCLC patients. CD133 was found to promote NSCLC tumorigenesis and mediated the expression of CXCR4. Furthermore, CD133/CXCR4 co-expression was found to be an independent prognostic factor as shown by univariate and multivariate Cox
\end{abstract}

Correspondence to: Professor Qiuping Zhang or Dr Jie Xiong, Department of Immunology, School of Basic Medical Sciences, Wuhan University, 185 Donghu Road, Wuchang, Wuhan, Hubei 430071, P.R. China

E-mail: qpzhang@whu.edu.cn

E-mail: jiexiong@whu.edu.cn

${ }^{*}$ Contributed equally

Abbreviations: NSCLC, non-small cell lung cancer; EMT, epithelialmesenchymal transition; CSC, cancer stem cell

Key words: non-small cell lung cancer, metastasis, CD133, CXCR4, epithelial-mesenchymal transition regression analysis, and was observed to regulate the expression of EMT-related molecules and transcriptional factors in NSCLC. In addition, our results showed that E-cadherin and Vimentin were simultaneously downregulated and upregulated, in $\mathrm{CD}_{133}{ }^{+} \mathrm{CXCR} 4{ }^{+} \mathrm{A} 549$ cells, respectively. While E-cadherin was upregulated and Vimentin was downregulated in metastatic NSCLC patients. Vimentin expression was also observed to have a positive correlation with CD133/CXCR4 co-expression in NSCLC patients and survival analysis results suggested that Vimentin high expression might be significantly associated with poor survival rates of the patients. Thus, these results suggest that the CD133/CXCR4/EMT axis may be a prognostic marker and may provide novel targets for combinational therapies in the treatment of NSCLC.

\section{Introduction}

Lung cancer is one of the increasingly potential causes of cancer deaths in both males and females worldwide (1). It was estimated that 595,690 Americans will die from cancer in 2016 , with $>27 \%$ being due to lung cancer (2). Cancer statistics in China indicated that an estimated 4,292,000 new cancer cases and 2,814,000 cancer deaths would occur in 2015, with lung cancer being the most common incident cancer and the leading cause of cancer death (3). The main lung cancer types include small cell lung cancer and non-small cell lung cancer (NSCLC), which together account for $\sim 87 \%$ of all lung cancer cases (4). Most patients with NSCLC are observed to be in advanced stages of cancer at diagnosis, however even when diagnosed at an early stage and treated surgically, cancer recurrence has been observed in patients, with spread to the lymph node, liver and other tissues.

Metastasis is the major cause of death in patients with NSCLC. The process in which immobile epithelial cells transform to invasive cell types in what has been termed as epithelial-mesenchymal transition (EMT), is increasingly being accepted as having an instrumental role in tumor metastasis (5). EMT is characterized by the loss of cell-cell 
adhesions, and the transition to spindle-like cells that are capable of invading the extracellular matrix (6). An increasing number of studies have demonstrated that cancer stem cell (CSC) molecule CD133 promotes tumor invasion and metastasis by inducing EMT processes (7-15).

Human CD133 is a five transmembrane single-chain glycoprotein that belongs to the prominin family containing two large extracellular and two small intracellular loops (16). Lee et al examined the effects of IL-6 on growth, EMT processes and metastatic ability of $\mathrm{CD}_{133^{+}}$and $\mathrm{CD} 133^{-}$cell subpopulations isolated from NSCLC cells. Of which they demonstrated the dual roles of IL- 6 in regulating growth of $\mathrm{CD}_{133}^{-}$and $\mathrm{CD} 133^{+}$subpopulations of lung cancer cells, as well as the positive regulation of EMT/metastasis increase by IL-6 in CD133+ cells, but not in CD133- cells (14). Some researchers have suggested that CSCs are present in both blood and tumor tissue of NSCLC patients, and that EMT-inducing transcriptional factors Bmil may play an important role in initiation and maintenance of CSCs and might be involved in vascular dissemination of NSCLC (12). Others have shown that elevated CD133 expression is the signature marker of EMT and CSC association in lung adenocarcinoma (17). Additionally, Bertolini et al showed that $\mathrm{CD} 133^{+} / \mathrm{CXCR}^{+} /$ EpCAM- lung cancer-initiating cells sustain metastasis and correlate with poor prognosis (18).

CXCR4, a seven-transmembrane $\mathrm{G}$ protein-coupled receptor, is a physiological receptor for stromal-derivedfactor-1 (19) which is highly expressed in various types of human tumors (20-24). A number of studies have demonstrated the vital role of CXCR4 in cancer cell survival, proliferation, invasion and metastasis, and that CXCR4 promotes EMT processes in various cancers (25-29), with $\mathrm{CD} 133^{+} \mathrm{CXCR} 4^{+}$ cells showing a stronger invasiveness capacity (18,30-33).

Although researchers have suggested a possible role for CD133 and CXCR4 in NSCLC metastasis, the precise relationship of CD133, CXCR4 and EMT processes are unclear and requires further investigation. Therefore, in this study, we detected the expression of CD133 and CXCR4 in NSCLC patients and investigated the effects of CD133 in NSCLC tumorigenesis. We further investigated the CXCR4/CD133 interaction and explored the possible molecular mechanisms by which CXCR4/CD133 regulates NSCLC metastasis via the EMT process.

\section{Materials and methods}

GEO databases analysis. Gene expression data were obtained from the publicly available GEO databases. We chose GSE10072, GSE40275 and GSE63459 to identify the expression signature of CD133 because these sets included normal $(n>30)$ and tumor samples $(n>30)$. Original values were normalized by application of $\log _{2}$ method and comparisons were performed by two-tailed Student's t-test for GSE10072 and GSE40275, and paired t-test for GSE63459. We chose GSE30219 because it was the largest lung cancer dataset to identify the correlation between CD133 and CXCR4 by two-tailed bivariate analysis.

NSCLC tissue samples. A total of 64 specimens was collected from patients with primary NSCLC surgical resection at
Renmin Hospital of Wuhan University from May 2012 to April 2015. Patients were followed-up by telephone until recurrence/metastasis or until May 2016. This study was carried out in accordance with the principles of the Helsinki declaration and approved by the ethics committee of the Renmin Hospital of Wuhan University.

NSCLC cell lines. A549 was obtained from American Type Culture Collection (ATCC) and maintained in Dulbecco's modified Eagle's medium (DMEM) with high glucose (Gibco, USA) supplemented with $10 \%$ fetal bovine serum (Hyclone, USA), containing $100 \mathrm{U} / \mathrm{ml}$ penicillin and $100 \mathrm{mg} / \mathrm{ml}$ streptomycin. A549 cells were cultured in a $5 \% \mathrm{CO}_{2}$ air incubator at $37^{\circ} \mathrm{C}$ and passaged using $0.25 \%$ trypsin-EDTA (Gibco) when they reached confluence.

Immunohistochemistry. Paraffin-embedded tissue sections were dewaxed and rehydrated, and antigen retrieval was performed by microwaving in $10 \mathrm{mM}$ sodium citrate buffer, $\mathrm{pH} 6.0$, for $20 \mathrm{~min}$. Sections were then incubated with $3 \%$ hydrogen peroxide for $30 \mathrm{~min}$ at room temperature to block endogenous peroxidase, then blocked in $10 \%$ normal goat serum for $0.5 \mathrm{~h}$. Immunostaining was performed by incubating with anti-CXCR4 (1:500, ab124824; Abcam Corp., UK), anti-CD133 (1:200, orb99113; Biorbyt Ltd., UK), anti-E cadherin (1:500, ab40772; Abcam Corp.) or anti-vimentin (1:500, ab92547; Abcam Corp.) at $4^{\circ} \mathrm{C}$ overnight. Slides were then washed in PBS and incubated with secondary antibody (anti-rabbit detection system; Boster, China) for $30 \mathrm{~min}$ at $37^{\circ} \mathrm{C}$. Staining was visualized with 3,3 -diaminobenzidine and counterstained with hematoxylin. The final score is the average of the percentage of stained area [scored as 0 (without staining), $1(<25 \%), 2(25-50 \%)$ and $3(>50 \%)]$ and intensity [scored as 0 (without staining), 1 (light yellow), 2 (yellow) and 3 (brown yellow)] of stained cells The expression levels were divided into low expression (mean score $<2$ ).

Magnetic cell sorting. A549 cells were washed and suspended in PBS at a maximal concentration of $1.0 \times 10^{8}$ cells. Cells were incubated at $4^{\circ} \mathrm{C}$ with $100 \mu \mathrm{l} \mathrm{FcR} \mathrm{blocking} \mathrm{reagent} \mathrm{and} 100 \mu \mathrm{l}$ CD133 Micro Beads (130-097-049, Miltenyi Biotec, Germany) for $30 \mathrm{~min}$. Cells were isolated into $\mathrm{CD} 133^{+}$and CD133- cells by using MS columns (130-042-201, Miltenyi Biotec) and LD columns (130-042-901, Miltenyi Biotec) according to the manufacturer's instructions.

Immunofluorescence assay. Cells were fixed and permeabilized with $2 \%$ paraformaldehyde and $0.5 \%$ Triton X-100. After overnight incubation with anti-CD133 (1:50, 18470-1-AP; Proteintech, USA), the specimens were rinsed thoroughly and treated with anti-goat antibodies (1:100, BA1032, Boster), respectively. Nuclei were stained using 0.3 $\mu \mathrm{M}$ DAPI (C1002, Beyotime Biotechnology, China). The digital images were then captured with a cooled CCD camera and processed with the help of photoshop (Adobe) software.

Clone formation assay. Cells (500 cells/well) were seeded in 6 -well plate and allowed to grow for 2 weeks in $37^{\circ} \mathrm{C}$ incubator. The cells were then washed twice with ice-cold PBS, fixed by methanol for $15 \mathrm{~min}$ and stained with Giemsa for $15 \mathrm{~min}$. The 
images of staining and clone spheres of cells were obtained by a charge coupled device camera.

Cell proliferation assay. Cells were seeded in a 96-well plate at the density of $1.0 \times 10^{4}$ cells per well for $48 \mathrm{~h}, 10 \mu \mathrm{l}$ CCK8 solution was added to each well at indicated times and incubated for another $2 \mathrm{~h}$. The absorbance of each well was obtained from PerkinElmer 2030 Victor X multi-label plate reader (Perkin-Elmer, Waltham, MA, USA) at $450 \mathrm{~nm}$.

SiRNA transfect assay. Cells were plated $24 \mathrm{~h}$ before transfection at $30-50 \%$ confluence, cells were transfected using Lipofectamine $^{\mathrm{TM}} 2000$ (Invitrogen, USA) with siRNA duplexes specific for human CD133 (Ribobio, China) or negative control (NC) siRNA. The following were the sequences of CD133 siRNA used in the study: sense-CUGGGAAGCU AUUUAAUAA; antisense-UUAUUAAAUAGCUUCCCAG. In addition, control was included where cells were treated with Lipofectamine 2000 alone. The siRNA experiment was carried out for $48 \mathrm{~h}$ to analyse the RNA expression level by quantitative RT-PCR and $72 \mathrm{~h}$ to analyse the protein expression level by western blot analysis.

Quantitative RT-PCR. Total RNA was extracted by using TRIzol (Invitrogen) according to the manufacturer's specifications and quantified by NanoDrop 2000 (Thermo Scientific, USA). Total $1 \mu \mathrm{g}$ RNA was reverse-transcribed to cDNA with random primers using the Promega reverse transcriptase kit (USA) according to manufacturer's protocol. To assess gene expression, cDNAs were amplified with the SYBR ${ }^{\circledR}$ Premix Ex Taq ${ }^{\text {TM }}$ II (Tli RNaseH Plus) (Takara, Japan) using the QuantStudio 6 Flex Real-Time PCR system (Life Technologies (AB and Invitrogen) USA]. The relative levels were calculated by the comparative $\mathrm{Ct}$ method $\left(2^{-\Delta \Delta \mathrm{Ct}}\right)$. The sequences of specific primers are shown in Table I.

Semi-quantitative RT-PCR. To assess gene expression, cDNAs were amplified with the $2 \mathrm{X}$ Ex $\mathrm{Taq}^{\mathrm{TM}}$ master mix (CWBio, China) using the Mycycler thermal cycler PCR system (Bio-Rad, USA). The expression of each gene was captured by Gel imaging system (BiD-1D/VILBER, France). The sequences of specific primers are shown in Table I.

Western blot analysis. Cells were washed with ice-cold PBS and lysed by RIPA buffer supplemented with protease inhibitor PMSF on ice. Total protein concentrations were measured by BCA kit (Thermo Scientific) and absorbance was measured by the PerkinElmer 2030 Victor X multi-label plate reader. The extracted proteins were separated by a $12 \%$ SDS-polyacrylamide gel electrophoresis and transferred to PVDF membranes (Millipore, USA). The membranes were blocked with $5 \%$ non-fat milk TBS-T $(0.1 \%$ Tween- 20 , $100 \mathrm{nM}$ Tris- $\mathrm{HCl}, 0.9 \% \mathrm{NaCl})$ and subsequently incubated with anti-CXCR4 (1:200, sc-6190; Santa Cruz, USA), antiCD133 (1:500, orb99113; Biorbyt Ltd.), anti-E-cadherin (1:10,000, ab40772; Abcam Corp.) or anti-vimentin (1:2,000, ab92547; Abcam Corp.) respectively, with gentle shaking at $4^{\circ} \mathrm{C}$ overnight. After washing three times, the membranes were incubated with HRP-conjugated secondary antibodies for $2 \mathrm{~h}$ at room temperature. The protein bands were visual-
Table I. The sequences of specific primers for PCR or qPCR in this study.

\begin{tabular}{ll}
\hline Gene & \multicolumn{1}{c}{ Primer sequences } \\
\hline s-actin & F: 5'-AGCGAGCATCCCCCAAAGTT-3' \\
& R: 5'-GGGCACGAAGGCTCATCATT-3' \\
CD133 & F: 5'-GCACTCTATACCAAAGCGTCAA-3' \\
& R: 5'-CACGATGCCACTTTCTCACT-3' \\
CXCR4 & F: 5'-ATCTGTGACCGCTTCTACCC-3' \\
& R: 5'-CGATGCTGATCCCAATGTAG-3' \\
E-cadherin & F: 5'-CGTAGCAGTGACGAATGTGG-3' \\
& R: 5'-CTGGGCAGTGTAGGATGTGA-3' \\
Vimentin & F: 5'-CGCCAACTACATCGACAAGG-3' \\
& R: 5'-GGCTTTGTCGTTGGTTAGCT-3' \\
Snail & F: 5'-TTTACCTTCCAGCAGCCCTACGA-3' \\
& R: 5'-GCCTTTCCCACTGTCCTCATCT-3' \\
Slug & F: 5'-TCAAGAAGCATTTCAACGCCTC-3' \\
& R: 5'-TGAGCTGAGGATCTCTGGTTGT-3' \\
Twist & F: 5'-AGCAACAGCGAGGAAGAGCC-3' \\
& R: 5'-CACAGCCCGCAGACTTCTTG-3' \\
\hline
\end{tabular}

$\mathrm{F}$, forward. R, reverse.

ized with ECL plus western blot analysis detection reagents (Thermo Scientific).

Transwell migration assay. A total of $5 \times 10^{4}$ cells was plated in the upper chamber, which had a $6.5-\mathrm{mm}$ diameter polycarbonate membrane with an $8-\mu \mathrm{m}$ pore size coated filter (Corning, USA). The lower chamber was filled with $600 \mu 1$ DMEM solution containing $10 \% \mathrm{FBS}$, which acted as a chemoattractant. After $24 \mathrm{~h}$ of incubation at $37^{\circ} \mathrm{C}$, non-migrated cells were removed with cotton swabs and migrated cells were fixed in methanol and stained with $0.1 \%$ crystal violet. Images were obtained by light microscope with a charge coupled device camera. Cells from four different fields were counted to obtain the migration rates.

Statistical analysis. All assays were performed in triplicates. Statistical analysis was performed using SPSS 19.0 for Windows or GraphPad Prism 5 software. Data are reported as means \pm SD. Statistical differences were analyzed by Student's t-test for data between control and treated groups, or a one-way analysis of variance (ANOVA) for data from multiple groups, with the level of significance set at $\mathrm{p}<0.05$.

\section{Results}

CD133 and CXCR4 are highly expressed in NSCLC patients with metastasis. To explore the effect of CD133 in lung cancer, we downloaded GSE10072, GSE40275 and GSE63459 to identify the expression of CD133 in lung cancer and normal lung tissues. These results showed that CD133 was highly expressed in lung cancer compared to normal lung tissues 

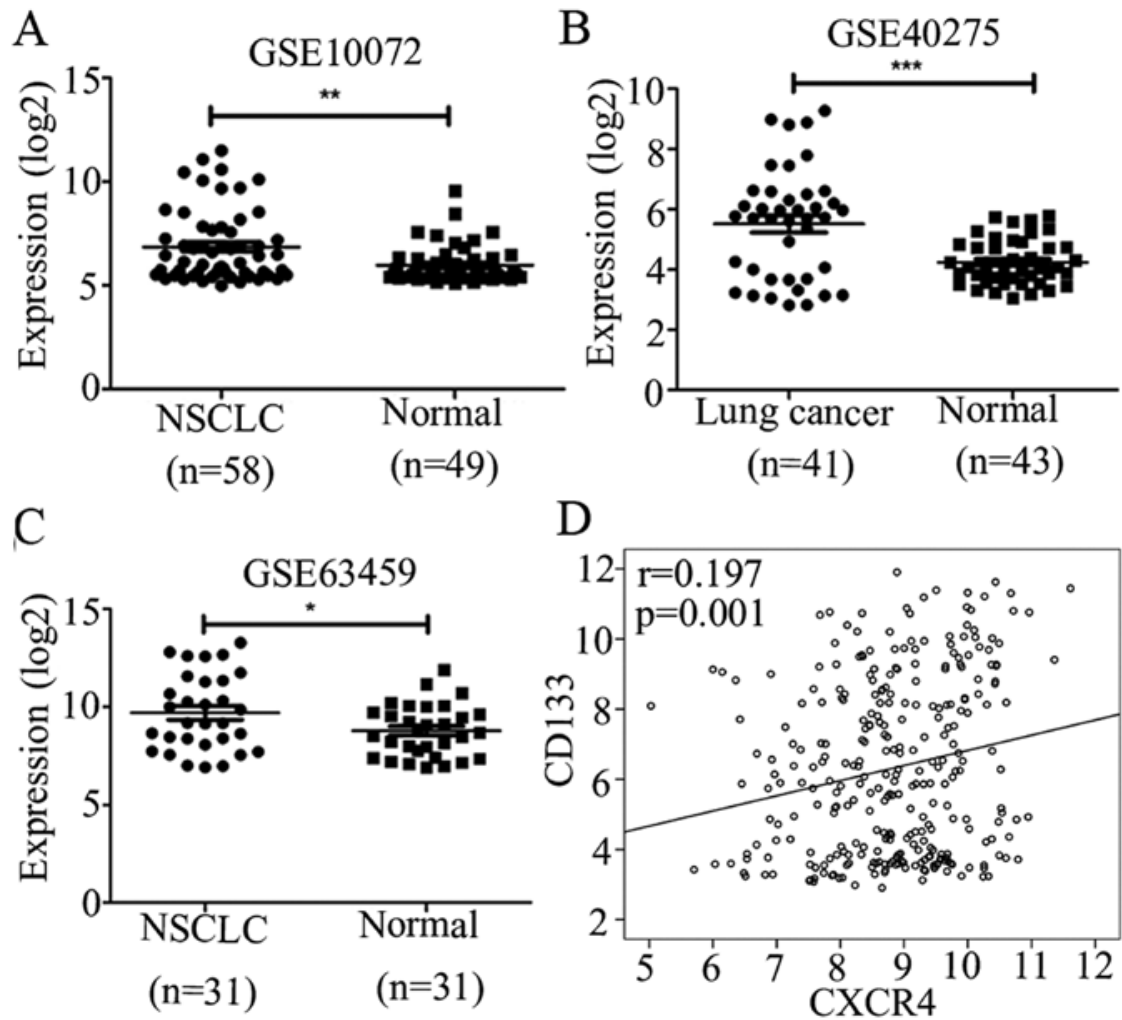

Figure 1. The expression of CD133 in lung cancer and normal lung tissues. Original values are presented as $\log _{2}$ ratios. Spots showing the level of CD133 mRNA in NSCLC and normal tissues. (A) GSE10072. (B) GSE40275. (C) GSE63459; ${ }^{*} \mathrm{p}<0.05,{ }^{* *} \mathrm{p}<0.01$ and ${ }^{* * * *} \mathrm{p}<0.001$. (D) Correlation between CD133 and CXCR4 in GSE30219.

(Fig. 1A-C), and we chose GSE30219, which is the largest dataset from GEO datasets to identify the correlation of CD133 and CXCR4, it showed that CD133 positively correlated with CXCR4 (Fig. 1D). Further, to investigate the role of CD133 and CXCR4 in NSCLC metastasis, we first detected the expression levels of CD133 and CXCR4 in 64 NSCLC patients by immunohistochemistry, the clinical and pathological characteristics of the patients are shown in Table II. These results showed that CD133 and CXCR4 levels significantly increased in NSCLC metastatic patients as compared to those in non-metastatic NSCLC patients (Fig. 2A). We categorized NSCLC patients with metastasis into low (34.8\%) and high group (75.6\%), and patients with non-metastasis into low $(65.2 \%)$ and high group (24.4\%) for CD133 expression (Fig. 2B), then we categorized NSCLC patients with metastasis into low (47.8\%) and high group (65.9\%) and patients with non-metastasis into low (52.2\%) and high group (34.1\%) for CXCR4 expression, respectively (Fig. 2C). The staining scores were $1.57 \pm 0.24$ in metastasis group and $0.95 \pm 0.14$ in non-metastasis group for CD133 expression (Fig. 2D), were $1.61 \pm 0.22$ in metastasis group and $1.17 \pm 0.17$ in non-metastasis group for CXCR4 expression (Fig. 2E), respectively.

These results show a significant association between CD133 and CXCR4 expression levels with NSCLC metastatic state in patients. A positive correlation between CD133 and CXCR4 expression was observed as shown in Fig. $2 \mathrm{~F}$ ( $\mathrm{r}=0.419$, $\mathrm{p}=0.001)$.

To further study the association between CD133/CXCR4 expression and cancer patient prognosis, we divided the patients into two groups which including CD133/CXCR4
Table II. The clinical and pathological characteristics of the patients.

Features

Patients $(n=64)$

$\begin{array}{ll}\text { Age (years) } & \\ \leq 65 & 15 \\ >65 & 49\end{array}$

Gender

Male 44

Female 20

T stage

$\mathrm{T} 2$

T3 14

T4

$\mathrm{N}$ stage

NO 36

N1

N2 21

M stage

M0

62

M1

Histology

Adenocarcinoma 39

Squamous carcinoma $\quad 25$ 
A

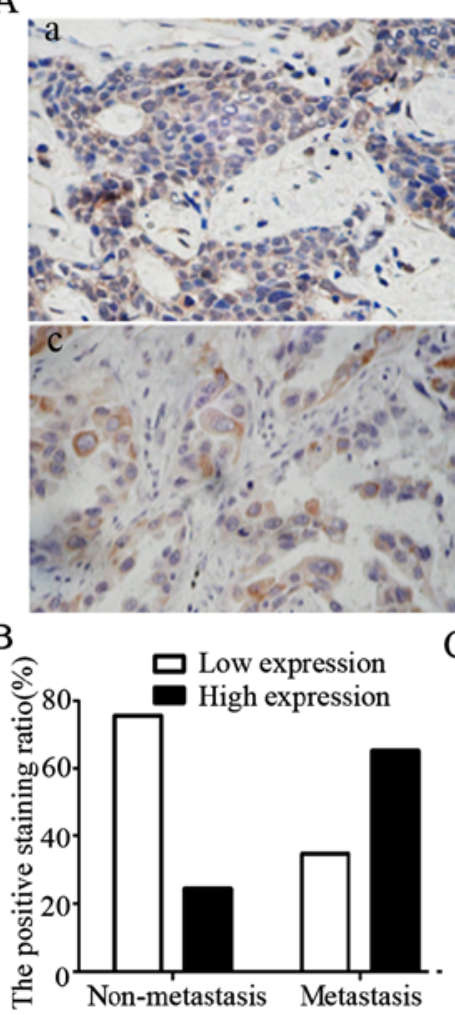

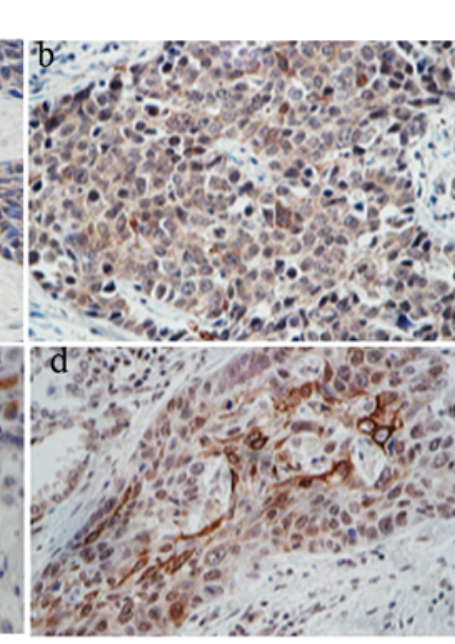

$\mathrm{C}$

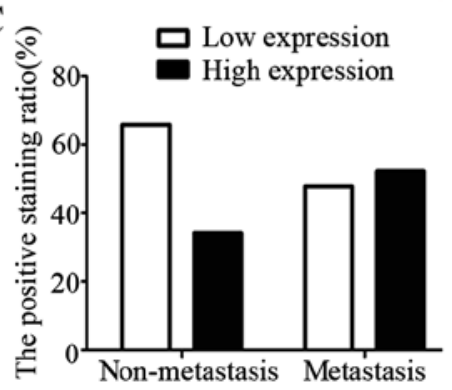

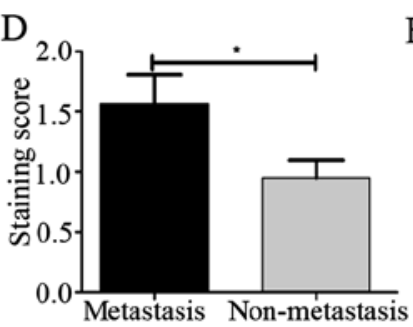

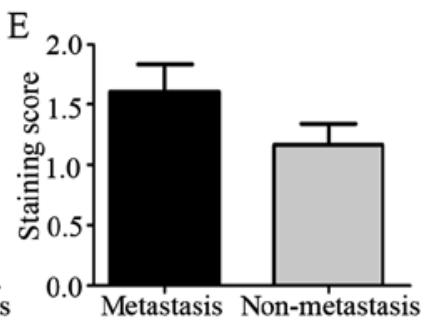

$\mathrm{F}$

Correlations

\begin{tabular}{|ll|r|r|}
\hline & & \multicolumn{1}{|c|}{ CD133 } & CXCR4 \\
\hline CXCR4 & Pearson Correlation & 1 & $0.419^{* *}$ \\
& Sig.(2-tailed) & 64 & 0.001 \\
& $\mathrm{~N}$ & 64 \\
\hline CD133 & Pearson Correlation & $0.419^{* *}$ & 1 \\
& Sig.(2-tailed) & 0.001 & 64 \\
& $\mathrm{~N}$ & 64 & 64 \\
\hline
\end{tabular}

G

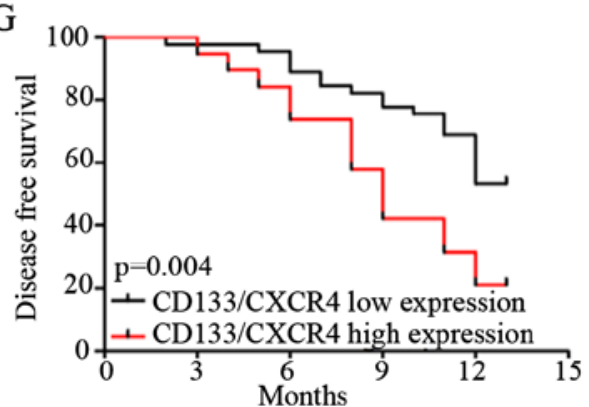

Figure 2. CD133 and CXCR4 are highly expressed in NSCLC patients with metastasis. (A) Representative images of immunohistochemistry staining (magnification, x400) of NSCLC patients. (a) CD133 low expression; (b) CD133 high expression; (c) CXCR4 low expression; (d) CXCR4 high expression. Positive staining ratio of CD133 (B)/CXCR4 (C) in metastatic and non-metastatic NSCLC patients. Positive staining score of CD133 (D)/CXCR4 (E) in metastatic and non-metastatic NSCLC patients. (F) Correlation results of CD133 and CXCR4. (G) Correlation analysis between CD133/CXCR4 co-expression and diseasefree survival (red and black curves indicate high and low CD133/CXCR4 co-expression groups of patient death, respectively; ${ }^{*} \mathrm{p}<0.05$ ).

Table III. Univariate and multivariate Cox regression analyses in NSCLC patients.

\begin{tabular}{|c|c|c|c|c|c|c|}
\hline \multirow[b]{2}{*}{ Variables } & \multicolumn{3}{|c|}{ Univariate model } & \multicolumn{3}{|c|}{ Multivariate model } \\
\hline & HR & $95 \% \mathrm{CI}$ of $\mathrm{HR}$ & P-value & HR & $95 \% \mathrm{CI}$ of $\mathrm{HR}$ & P-value \\
\hline Gender & 0.858 & $0.422-1.745$ & 0.672 & 0.924 & $0.385-2.218$ & 0.860 \\
\hline Age & 1.007 & $0.969-1.046$ & 0.729 & 0.999 & $0.952-1.048$ & 0.965 \\
\hline Histology & 0.754 & $0.388-1.463$ & 0.404 & 1.363 & $0.532-3.488$ & 0.519 \\
\hline T stage & 1.475 & $0.953-2.282$ & 0.081 & 1.514 & $0.917-2.499$ & 0.105 \\
\hline $\mathrm{N}$ stage & 1.659 & $1.182-2.329$ & 0.003 & 1.672 & $1.107-2.526$ & 0.014 \\
\hline M stage & 2.610 & $0.617-11.047$ & 0.192 & 2.291 & $0.412-12.732$ & 0.343 \\
\hline CD133/CXCR4 co-expression & 2.722 & $1.400-5.294$ & 0.003 & 2.480 & $1.077-5.709$ & 0.033 \\
\hline
\end{tabular}

FDR, false discovery rate; HR, hazards ratio; CI, confidence interval.

high expression (score $\geq 4$ ) and low expression (score $<4$ ) group according to the immunohistochemistry staining score average (stained area and intensity) and we analyzed the correlation between CD133/CXCR4 co-expression and cancer patient disease-free survival, and we observed that patients with high expression of both CD133 and CXCR4 presented a significantly poor survival rate (Fig. 2G). Using univariate and multivariate Cox regression models, we also observed that $\mathrm{N}$ stage and CD133/CXCR4 co-expression correlated with a shorter disease-free survival. These results therefore suggest that CD133/CXCR4 co-expression may be a potential independent predictor of NSCLC patient survival (Table III).

CD133 promotes NSCLC tumorigenesis. To investigate the role of CD133 in NSCLC tumorigenesis, CD133 ${ }^{+}$and CD133 A549 cells were sorted by magnetic cell sorting and verified by immunofluorescence assay. Our results indicated that CD133 glycoproteins were generally expressed in the $\mathrm{CD}_{133^{+}}$as compared to the CD133- groups (Fig. 3A). Furthermore, colony 

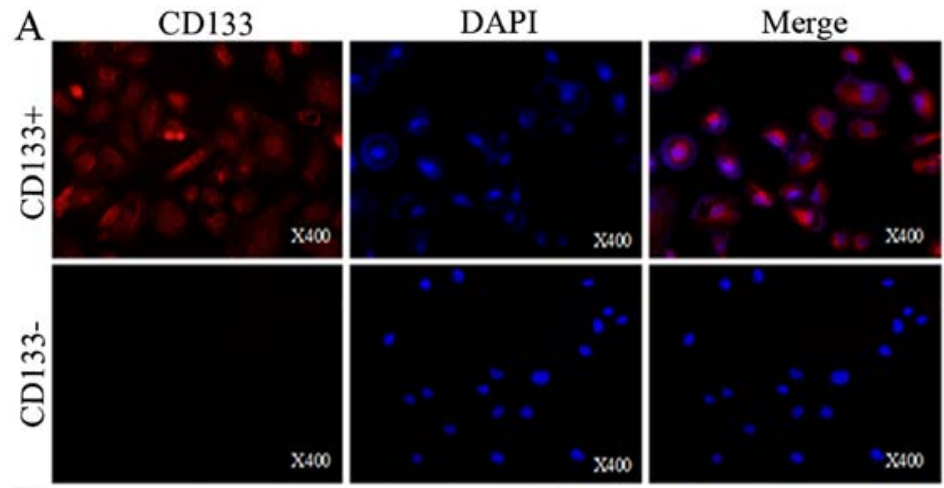

B

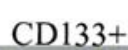

CD133-
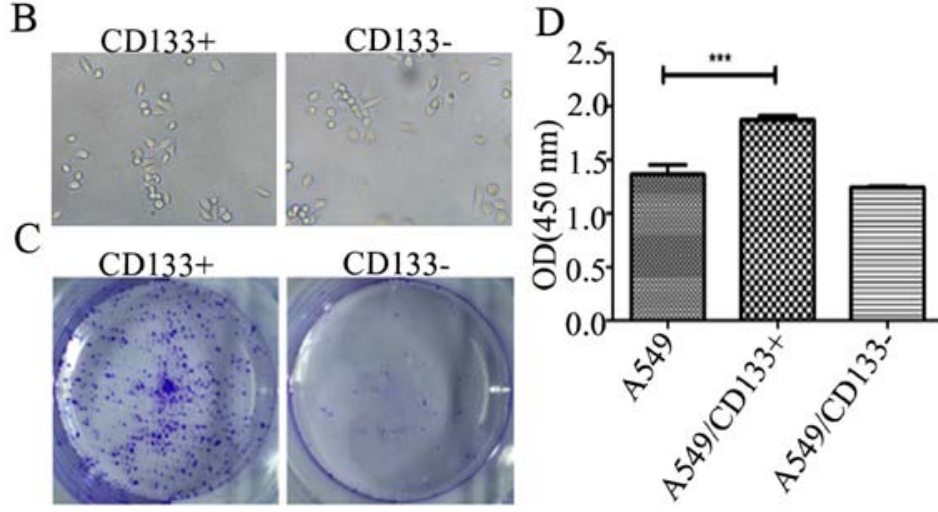

Figure 3. CD133 enhances A549 cells proliferation. (A) Effectiveness of magnetic cell sorting was verified by immunofluorescence assay (magnification, $\mathrm{x} 400$ ). Red and blue indicate CD133 expression and cell nucleus, respectively. (B) Formation of colonies by A549 cell lines after 2-week incubation. (C and D) Cell proliferation capacity was determined in A549 cells, CD133 ${ }^{+}$A549 cells and CD133-A549 cells by CCK8 assays.

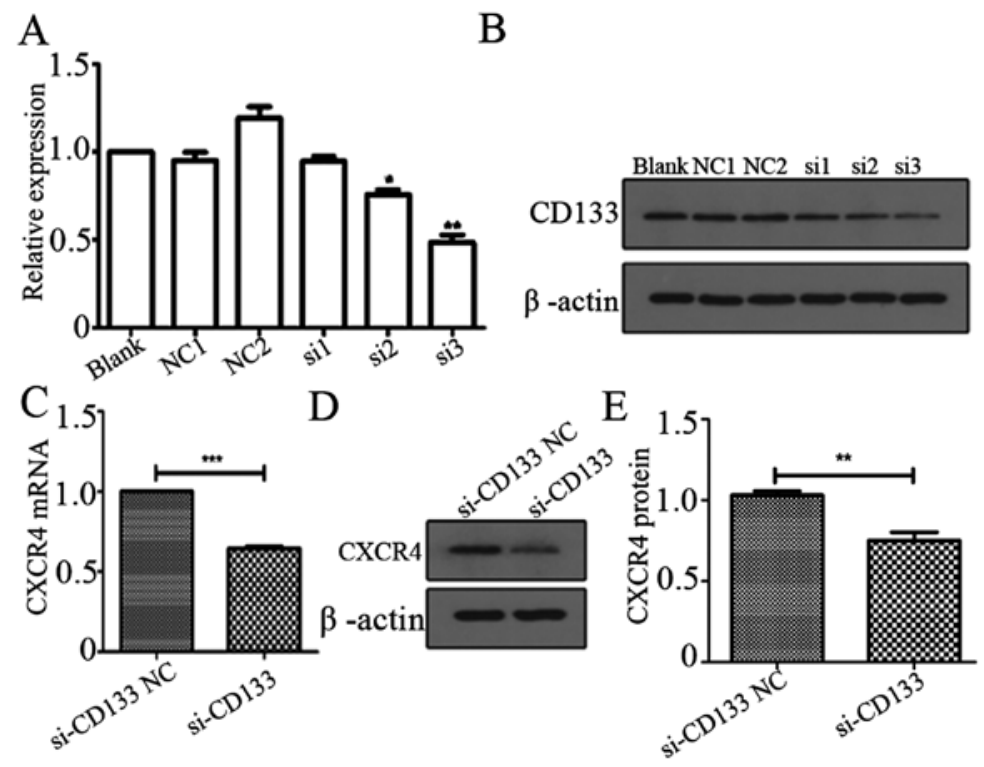

Figure 4. CXCR4 is upregulated by CD133. Silencing effectiveness of CD133 siRNA was verified by qPCR (A) and western blot analysis (B). (C) Expression of CXCR4 after treatment with CD133 siRNA (50 nM, 48 h) by qPCR analysis. (D) Western blot analysis for the expression of CXCR4 after treatment with CD133 siRNA (50 nM, $72 \mathrm{~h}$ ). $\beta$-actin as a loading control. (E) The statistics of (D). All experiments were performed in triplicate. ${ }^{* * * *} \mathrm{p}<0.001$.

formation assay and CCK8 assay results revealed that $\mathrm{CD}_{133^{+}}$ cells had a significant growth advantage over the control cells as shown in Fig. 3B and C.

CD133 mediates the expression of CXCR4 in NSCLC. We previously found that CD133 and CXCR4 were highly expressed in A549 cells, so here to investigate the effect of CD133 on
CXCR4 expression, we first demonstrated that CD133 mRNA and protein levels were significantly inhibited by CD133 siRNA (Fig. 4A and B). We then observed the effect of CD133 on the mRNA and protein expression of CXCR4 by CD133 silencing using CD133 siRNA (si3). We observed that CXCR4 mRNA and protein levels were significantly downregulated as a result of CD133 silencing (Fig. 4C-E). Taken together, these results 


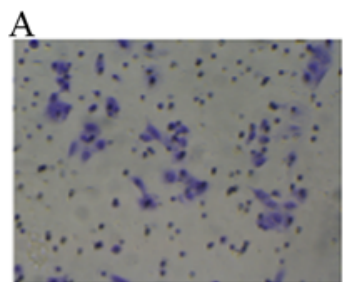

CD133-CXCR4+

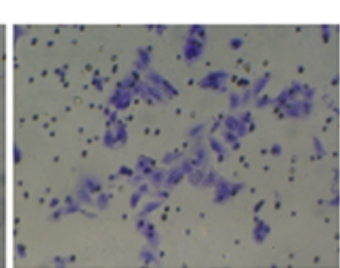

CD133+CXCR4-

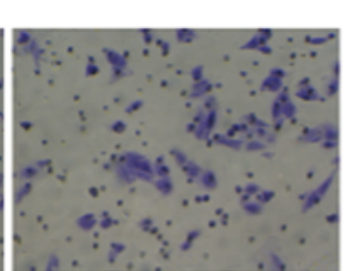

CD133+CXCR4

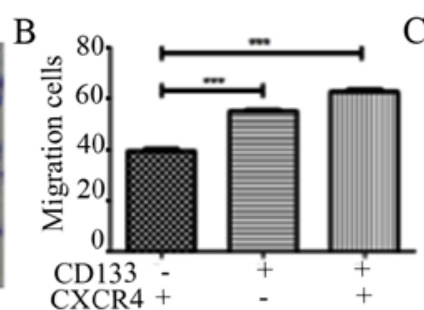

CXCR4 +
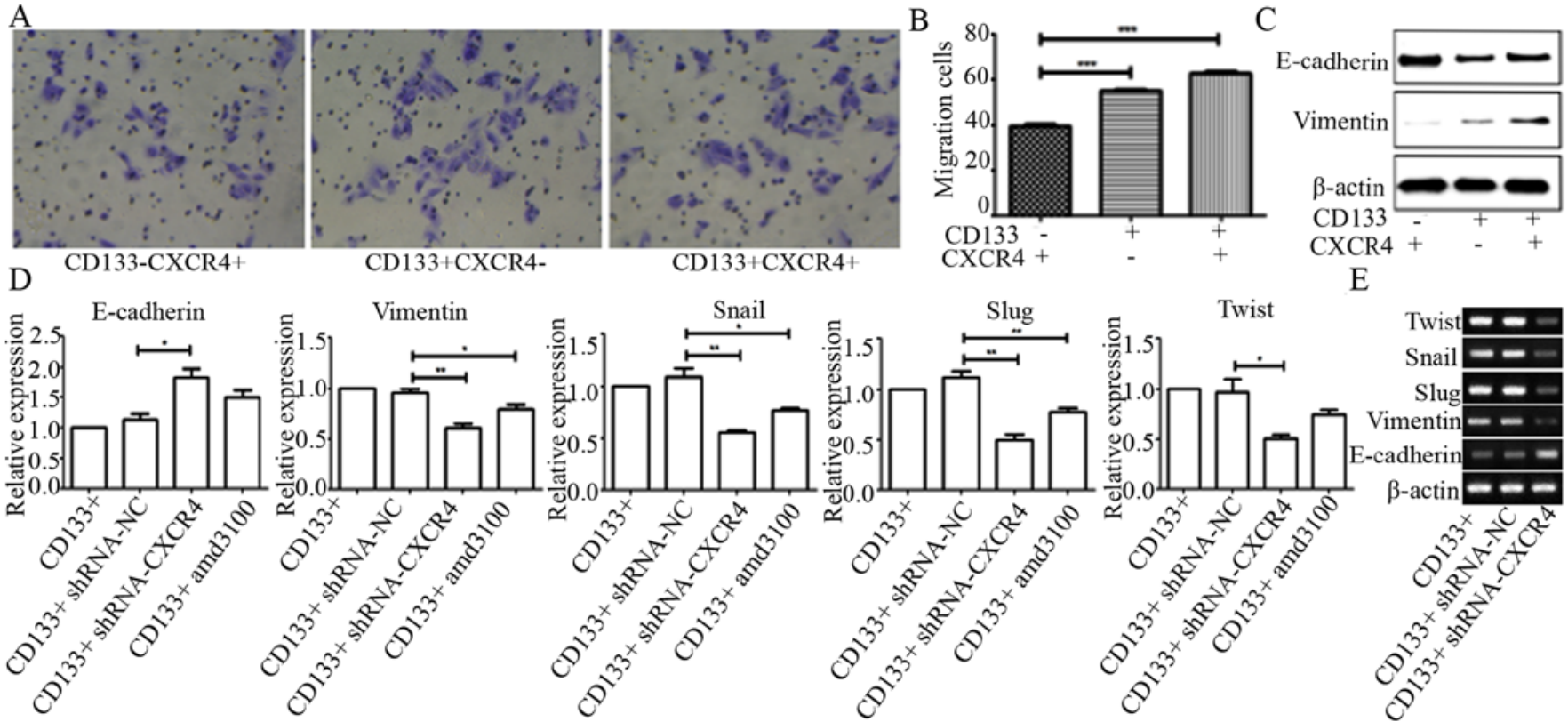

$\mathrm{E}$

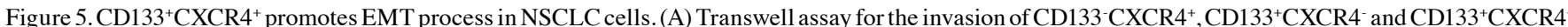
cells. (B) Western blot assay for the expression of E-cadherin and Vimentin in CD133 CXCR4 ${ }^{+}, \mathrm{CD} 133^{+} \mathrm{CXCR} 4^{-}$and CD133 ${ }^{+} \mathrm{CXCR} 4^{+}$cells. $(\mathrm{C}) \mathrm{qPCR}$ for the expression of E-cadherin, Vimentin, Snail, Slug and Twist in A549/CD133 ${ }^{+}$, A549/CD133+ shRNA-NC, A549/CD133 ${ }^{+}$shRNA-CXCR4 and A549/CD133 ${ }^{+}$with amd3100. (D and E) RT-PCR for the expression of E-cadherin, Vimentin, Snail, Slug and Twist in A549/CD133 ${ }^{+}$, A549/CD133 ${ }^{+}$shRNA-NC and A549/CD133 ${ }^{+}$ shRNA-CXCR4 cells.

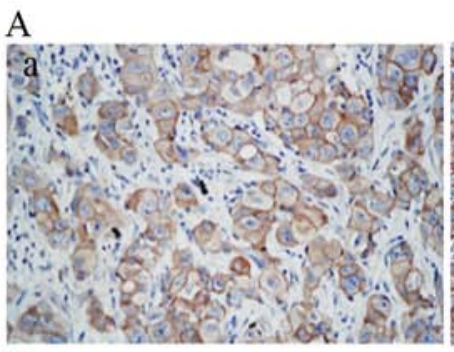

B

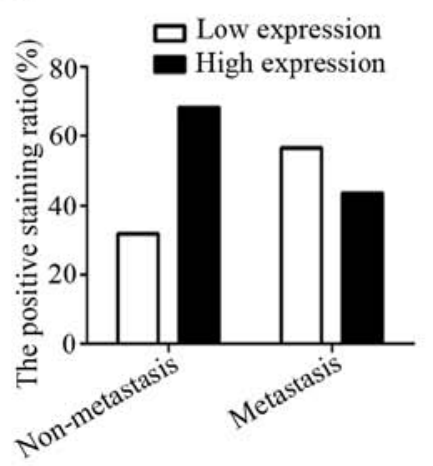

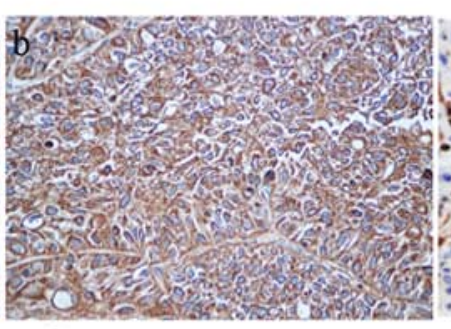

$\mathrm{C}$

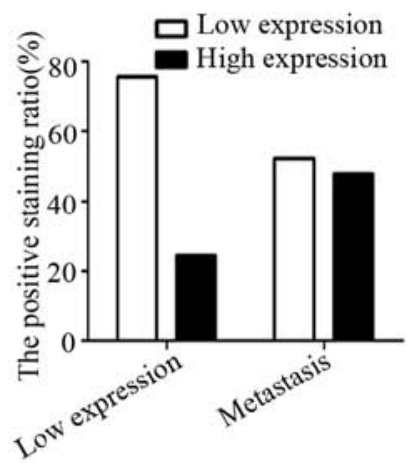

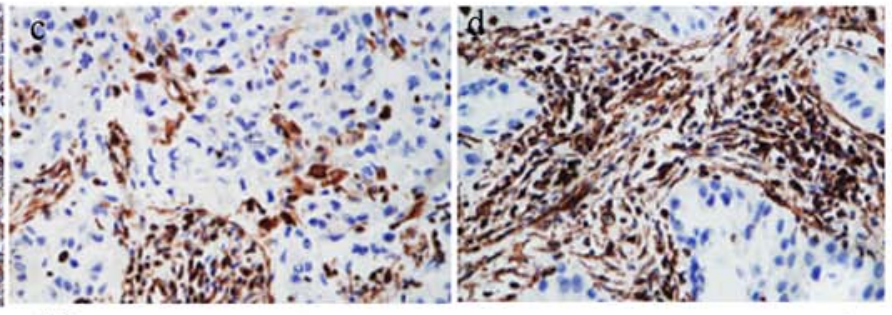

$\mathrm{D}$

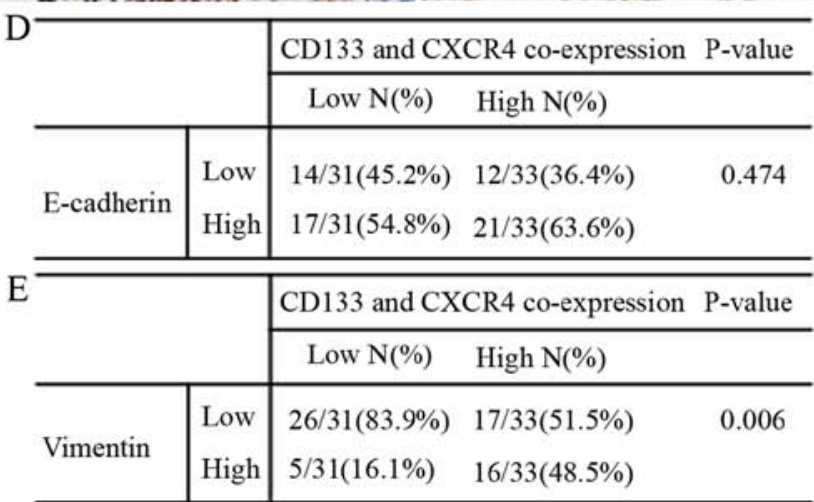

Figure 6. Vimentin is positively associated with CD133/CXCR4 co-expression. (A) Representative images of immunohistochemistry staining (magnification, $\mathrm{x} 400$ ) of NSCLC patients. (a) E-cadherin low expression; (b) E-cadherin high expression; (c) Vimentin low expression; (d) Vimentin high expression. Positive staining ratio of E-cadherin (B)/Vimentin (C) in metastatic and non-metastatic NSCLC patients. Correlation analysis for E-cadherin (D) or Vimentin (E) with CD133/CXCR4 co-expression in NSCLC patients.

demonstrate that CD133 might play a significant role in the CXCR4 regulation.

$C D 133^{+} C X C R 4^{+}$promotes EMT process in NSCLC. To investigate whether CXCR4 plays a vital role in CD133-induced
EMT processes, we first sorted A549 cells into CD133 $\mathrm{CXCR}^{+}, \mathrm{CD}_{133}{ }^{+} \mathrm{CXCR} 4{ }^{-}$and $\mathrm{CD} 133^{+} \mathrm{CXCR} 4^{+}$groups Our results showed remarkably higher migration rates in $\mathrm{CD} 33^{+} \mathrm{CXCR}^{+}$group as compared to the $\mathrm{CD} 133^{-} \mathrm{CXCR} 4^{+}$ group as revealed by Transwell migration assay (Fig. 5A 
and B). We also detected the differential protein expression of E-cadherin and Vimentin in CD133-CXCR4 ${ }^{+}, \mathrm{CD}^{-} 33^{+} \mathrm{CXCR} 4$ and $\mathrm{CD} 133{ }^{+} \mathrm{CXCR} 4^{+}$groups by western blot analysis, and results showed that $E$-cadherin was decreased, while Vimentin was increased in $\mathrm{CD}_{133}{ }^{+} \mathrm{CXCR} 4^{+}$as compared to the $\mathrm{CD} 133$ $\mathrm{CXCR}^{+}$(Fig. 5C).

We then went a step further and detected the expression of EMT-related molecules (E-cadherin, Vimentin) and transcriptional factors (Snail, Slug and Twist) in CD133 ${ }^{+}$549 cells in the presence of CXCR4 inhibitors (amd3100) or CXCR4 siRNA. Our findings indicated that E-cadherin was significantly upregulated, while Vimentin, Snail, Slug and Twist were significantly downregulated as a result of CXCR4 silencing or presence of CXCR4 inhibitors (Fig. 5D and E). These results demonstrate that $\mathrm{CD} 133$ potentially regulates the expression of CXCR4 in inducing EMT processes.

Expression of Vimentin was positively associated with CD133/CXCR4 co-expression. To investigate the role of CD133, CXCR4 and EMT-related molecules (E-cadherin and Vimentin) in NSCLC metastasis, we detected the expression levels of CD133 and CXCR4, and investigated the correlative expression of EMT-related molecules (E-cadherin and Vimentin) with CD133/CXCR4 co-expression in 64 NSCLC patients by immunohistochemistry. Our results revealed that there was a significant increase in the Vimentin levels, while the expression levels of E-cadherin decreased as shown in Fig. 6A. NSCLC metastatic patients were categorized as either low $(56.5 \%)$ or high $(43.5 \%)$ E-cadherin expression groups and non-metastatic patients were categorized as either low (31.6\%) or high $(68.4 \%)$ E-cadherin expression groups. In terms of Vimentin expression, NSCLC metastatic patients were categorized as either low (52.2\%) or high (47.8\%) expression groups, while non-metastatic patients were further categorized as either low (75.6\%) or high (24.4\%) expression groups (Fig. 6B and $\mathrm{C}$ ).

We found E-cadherin expression did not correlate with CD133/CXCR4 co-expression (Fig. 6D), while Vimentin had a positive correlative expression with CD133/CXCR4 co-expression in NSCLC patients $(p=0.006$, Fig. 6E). Taken together these results suggest that Vimentin is involved in CD133/CXCR4 induced NSCLC metastasis.

\section{Discussion}

Lung cancer is one of the leading causes of cancer mortality worldwide. Being the most dominating type of lung cancer, NSCLC cases are being diagnosed at more advanced stages and hence has been associated with a poor prognosis in patients (4). So far, little success has been made in significantly improving patient survival rates, as the 5-year survival rates have remained around $18 \%(34,35)$.

The main challenges for the NSCLC patient prognosis are tumor metastasis and chemoresistance. Metastasis is a multi-step process, which begins when primary tumor cells break away from their neighboring cells invading the basement membrane, subsequently entering the circulation, either directly or via the lymphatics and eventually homing in distant organs. Tumor cells that successfully adapt to the new microenvironments proliferate from micro-metastases into clinical detectable metastatic tumor (36). The CSCs in tumor microenvironment are vital for the acquisition of the metastatic potential as they initiate, drive carcinogenesis and differentiation. In essence contributing to tumor cellular heterogeneity through the deregulation of the self-renewal processes, thus CSC populations may be a risk factor for carcinogenesis (37).

Previous studies have identified a number of cellular markers which include CD133 (38-40) and CXCR4 (41,42), that may contribute to the properties exhibited by lung CSCs. Chemokines are a family of small secreted proteins of $\sim 70-80$ amino acids. CXCR4 is an $\alpha$-chemokine receptor specific for the CXCL12. Unlike other chemokine receptors that have several ligands, CXCL12 is the only known specific legend for the receptor CXCR4 (43). We previously reported that CXCR4 was upregulated in NSCLC patients, and that specific downregulation of CXCR4 inhibited cell growth, invasiveness and migration. Additionally, we also reported that MMP2 and MMP9 could be upregulated after treatment with CXCL12 in A549 cells $(20,21)$, and that CXCR4 was highly expressed in chemo-resistant NSCLC patients and induced anti-apoptosis to cisplatin in a CYP1B1 manner (data not shown).

In this study, we found that CD133 and CXCR4 are highly expressed in NSCLC metastatic patients as compared to nonmetastatic patients. We further used Cox regression analysis to verify whether CD133/CXCR4 could be a potential prognostic marker for NSCLC patients, of which univariate Cox regression results indicated that the $\mathrm{N}$ stage, $\mathrm{M}$ stage and CD133/CXCR4 co-expression significantly associated with the patient prognosis. Moreover, multivariate Cox regression results indicated that only the $\mathrm{N}$ stage and CD133/CXCR4 co-expression significantly correlated with the patient prognosis, with a CD133/CXCR4 co-expression hazards ratio of 2.48 , thus suggesting that CD133/CXCR4 may be an independent prognostic marker in NSCLC patients. In the same line, other researchers have also shown that CD133/CXCR4 co-expression is highly associated with poor prognosis in patients (18). We also investigated the role of CD133 in promoting NSCLC tumorigenesis as a potential CSCs marker, as in some types of tumors, the stem cells exert increased proliferation $(44,45)$, furthermore, CD133-positive cells showed proliferative characters in various tumors (46-48), which are consistent with our results, so these results indicated that CXCR4 might be modulated in part via CD133, as shown by the CXCR4 mRNA and protein expression downregulation upon CD133 silencing, but, CXCR4 silencing did not influence the CD133 expression after transfected with siCXCR4 or siNC for $48 \mathrm{~h}$ in A549 cells.

In recent years, EMT has emerged as a potential mechanism underlying cancer progression and metastasis. EMT processes are accompanied by profound changes in cell characteristics, which enable the epithelial cell to detach from tight junctions, change their shape and polarity, delaminate and migrate. As a result, mesenchymal cells exhibit a front-rear polarity, become flat and spindle-shaped, and become loosely associated with neighboring mesenchymal cells (49). We therefore also explored the role of CD133/CXCR4 as a CSCs marker in mediating EMT processes and observed that $\mathrm{CD} 133^{+} \mathrm{CXCR} 4^{+}$ cells had a stronger migration ability accompanied by a lower E-cadherin expression and a higher Vimentin expression as 
compared to $\mathrm{CD} 33^{-} \mathrm{CXCR}^{+}{ }^{+}$and $\mathrm{CD} 133^{+}{ }^{+} \mathrm{CXCR} 4^{-} \mathrm{A} 549$ cells. Knapp et al also used immunohistochemical analysis of CXCR4 and E-cadherin on a melanoma tissue microarray which included 110 primaries, 73 local/regional metastatic and 44 distant metastatic patients, and observed that there was no significant E-cadherin expression (50).

Furthermore, we detected the expression of EMT-related molecules (E-cadherin, Vimentin) and transcription factors (Snail, Slug and Twist) after CXCR4 silencing or inhibition by a CXCR4 antagonist. Our results showed that E-cadherin was upregulated, while Vimentin, Snail, Slug and Twist were downregulated as a result of CXCR4 knockdown or inhibition, thus suggesting a potential role for CXCR4 in regulating EMT processes.

In addition, Vimentin expression was observed to have a positive correlation with CD133/CXCR4 co-expression in NSCLC patients and survival analysis results suggested that Vimentin high expression might be significantly associated with poor patients' survival rates, thus suggesting that Vimentin may be involved in CD133/CXCR4 induced NSCLC metastasis. Vimentin expressing metastatic lung cancer cells have been reported to be more motile and invasive while acting via a VAV2-Rac1 pathway to control focal adhesion kinase activity (51). In conclusion, this study shows that CXCR4 is involved in CD133-induced EMT processes in NSCLC. Thus, the CD133/CXCR4/EMT axis could be used as a potential therapeutic target in NSCLC metastasis.

\section{Acknowledgements}

This study was supported by National Natural Science Foundation of China (nos. 81270607 and 81541027) and Natural Science Foundation of Hubei (nos. 2014CFA070 and 2015CFB653).

\section{References}

1. Miller KD, Siegel RL, Lin CC, Mariotto AB, Kramer JL, Rowland JH, Stein KD, Alteri R and Jemal A: Cancer treatment and survivorship statistics, 2016. CA Cancer J Clin 66: 271-289, 2016.

2. Siegel RL, Miller KD and Jemal A: Cancer statistics, 2016. CA Cancer J Clin 66: 7-30, 2016.

3. Chen W, Zheng R, Baade PD, Zhang S, Zeng H, Bray F, Jemal A, Yu XQ and He J: Cancer statistics in China, 2015. CA Cancer J Clin 66: 115-132, 2016.

4. Torre LA, Bray F, Siegel RL, Ferlay J, Lortet-Tieulent J and Jemal A: Global cancer statistics, 2012. CA Cancer J Clin 65 87-108, 2015

5. Chaffer CL and Weinberg RA: A perspective on cancer cell metastasis. Science 331: 1559-1564, 2011.

6. Thiery JP, Acloque H, Huang RY and Nieto MA: Epithelialmesenchymal transitions in development and disease. Cell 139: 871-890, 2009.

7. Cai C, Yu JW, Wu JG, Lu RQ, Ni XC, Wang SL and Jiang BJ: CD133 promotes the invasion and metastasis of gastric cancer via epithelial-mesenchymal transition. Zhonghua Wei Chang Wai Ke Za Zhi 16: 662-667, 2013 (In Chinese).

8. Bock C, Kuhn C, Ditsch N, Krebold R, Heublein S, Mayr D, Doisneau-Sixou S and Jeschke U: Strong correlation between $\mathrm{N}$-cadherin and CD133 in breast cancer: Role of both markers in metastatic events. J Cancer Res Clin Oncol 140: 1873-1881, 2014.

9. Ding Q, Miyazaki Y, Tsukasa K, Matsubara S, Yoshimitsu M and Takao S: CD133 facilitates epithelial-mesenchymal transition through interaction with the ERK pathway in pancreatic cancer metastasis. Mol Cancer 13: 15, 2014.
10. Nomura A, Banerjee S, Chugh R, Dudeja V, Yamamoto M, Vickers SM and Saluja AK: CD133 initiates tumors, induces epithelial-mesenchymal transition and increases metastasis in pancreatic cancer. Oncotarget 6: 8313-8322, 2015.

11. Long H, Xiang T, Qi W, Huang J, Chen J, He L, Liang Z, Guo B, Li Y, Xie R, et al: $\mathrm{CD} 133^{+}$ovarian cancer stem-like cells promote non-stem cancer cell metastasis via CCL5 induced epithelialmesenchymal transition. Oncotarget 6: 5846-5859, 2015.

12. Koren A, Rijavec M, Kern I, Sodja E, Korosec P and Cufer T: BMI1, ALDH1A1, and CD133 transcripts connect epithelialmesenchymal transition to cancer stem cells in lung carcinoma. Stem Cells Int 2016: 9714315, 2016.

13. Latorre E, Carelli S, Raimondi I, D'Agostino V, Castiglioni I, Zucal C, Moro G, Luciani A, Ghilardi G, Monti E, et al: The ribonucleic complex HuR-MALAT1 represses CD133 expression and suppresses epithelial-mesenchymal transition in breast cancer. Cancer Res 76: 2626-2636, 2016.

14. Lee SO, Yang X, Duan S, Tsai Y, Strojny LR, Keng P and Chen Y: IL-6 promotes growth and epithelial-mesenchymal transition of CD133+ cells of non-small cell lung cancer. Oncotarget 7: 6626-6638, 2016

15. Moon Y, Kim D, Sohn H and Lim W: Effect of CD133 overexpression on the epithelial-to-mesenchymal transition in oral cancer cell lines. Clin Exp Metastasis 33: 487-496, 2016.

16. Fargeas CA, Florek M, Huttner WB and Corbeil D: Characterization of prominin-2, a new member of the prominin family of pentaspan membrane glycoproteins. J Biol Chem 278: 8586-8596, 2003.

17. Sowa T, Menju T, Sonobe M, Nakanishi T, Shikuma K, Imamura N, Motoyama H, Hijiya K, Aoyama A, Chen F, et al: Association between epithelial-mesenchymal transition and cancer stemness and their effect on the prognosis of lung adenocarcinoma. Cancer Med 4: 1853-1862, 2015.

18. Bertolini G, D'Amico L, Moro M, Landoni E, Perego P, Miceli R, Gatti L, Andriani F, Wong D, Caserini R, et al: Microenvironment-modulated metastatic CD $133^{+} / \mathrm{CXCR}^{+} /$ EpCAM- lung cancer-initiating cells sustain tumor dissemination and correlate with poor prognosis. Cancer Res 75: 3636-3649, 2015.

19. Teicher BA and Fricker SP: CXCL12 (SDF-1)/CXCR4 pathway in cancer. Clin Cancer Res 16: 2927-2931, 2010.

20. Dai X, Mao Z, Huang J, Xie S and Zhang H: The CXCL12/ CXCR4 autocrine loop increases the metastatic potential of non-small cell lung cancer in vitro. Oncol Lett 5: 277-282, 2013.

21. Xie S, Zeng W, Fan G, Huang J, Kang G, Geng Q, Cheng B, Wang W and Dong P: Effect of CXCL12/CXCR4 on increasing the metastatic potential of non-small cell lung cancer in vitro is inhibited through the downregulation of CXCR4 chemokine receptor expression. Oncol Lett 7: 941-947, 2014.

22. Nikzaban M, Hakhamaneshi MS, Fakhari S, Sheikhesmaili F, Roshani D, Ahsan B, Kamali F and Jalili A: The chemokine receptor CXCR4 is associated with the staging of gastric cancer. Adv Biomed Res 3: 16, 2014.

23. Okuyama Kishima M, de Oliveira CE, Banin-Hirata BK, LosiGuembarovski R, Brajão de Oliveira K, Amarante MK and Watanabe MA: Immunohistochemical expression of CXCR4 on breast cancer and its clinical significance. Anal Cell Pathol (Amst) 2015: 891020, 2015.

24. Teng F, Tian WY, Wang YM, Zhang YF, Guo F, Zhao J, Gao C and Xue FX: Cancer-associated fibroblasts promote the progression of endometrial cancer via the SDF-1/CXCR4 axis. J Hematol Oncol 9: 8, 2016

25. Hu TH, Yao Y, Yu S, Han LL, Wang WJ, Guo H, Tian T, Ruan ZP, Kang XM, Wang J, et al: SDF-1/CXCR4 promotes epithelialmesenchymal transition and progression of colorectal cancer by activation of the Wnt $/ \beta$-catenin signaling pathway. Cancer Lett 354: 417-426, 2014.

26. Li X, Li P, Chang Y, Xu Q, Wu Z, Ma Q and Wang Z: The SDF-1/CXCR4 axis induces epithelial-mesenchymal transition in hepatocellular carcinoma. Mol Cell Biochem 392: 77-84, 2014.

27. Lv B, Yang X, Lv S, Wang L, Fan K, Shi R, Wang F, Song H, Ma X, Tan X, et al: CXCR4 signaling induced epithelial-mesenchymal transition by PI3K/AKT and ERK pathways in glioblastoma. Mol Neurobiol 52: 1263-1268, 2015.

28. Yang P, Wang G, Huo H, Li Q, Zhao Y and Liu Y: SDF-1/CXCR4 signaling up-regulates survivin to regulate human sacral chondrosarcoma cell cycle and epithelial-mesenchymal transition via ERK and PI3K/AKT pathway. Med Oncol 32: 377, 2015. 
29. Liao A, Shi R, Jiang Y, Tian S, Li P, Song F, Qu Y, Li J, Yun H and Yang X: SDF-1/CXCR4 axis regulates cell cycle progression and epithelial-mesenchymal transition via up-regulation of survivin in glioblastoma. Mol Neurobiol 53: 210-215, 2016.

30. Zhang SS, Han ZP, Jing YY, Tao SF, Li TJ, Wang H, Wang Y, Li R, Yang Y, Zhao X, et al: CD133(+)CXCR4(+) colon cancer cells exhibit metastatic potential and predict poor prognosis of patients. BMC Med 10: 85, 2012

31. Silinsky J, Grimes C, Driscoll T, Green H, Cordova J, Davis NK, Li L and Margolin DA: CD $133^{+}$and CXCR4 ${ }^{+}$colon cancer cells as a marker for lymph node metastasis. J Surg Res 185: 113-118, 2013.

32. Li XF, Guo XG, Yang YY and Liu AY: Effect of CXCR4 and CD133 co-expression on the prognosis of patients with stage II-III colon cancer. Asian Pac J Cancer Prev 16: 1073-1076, 2015.

33. Lu C, Xu F, Gu J, Yuan Y, Zhao G, Yu X and Ge D: Clinical and biological significance of stem-like CD133(+)CXCR4(+) cells in esophageal squamous cell carcinoma. J Thorac Cardiovasc Surg 150: 386-395, 2015.

34. Siegel R, Naishadham D and Jemal A: Cancer statistics, 2013. CA Cancer J Clin 63: 11-30, 2013.

35. DeSantis CE, Lin CC, Mariotto AB, Siegel RL, Stein KD, Kramer JL, Alteri R, Robbins AS and Jemal A: Cancer treatment and survivorship statistics, 2014. CA Cancer J Clin 64: 252-271, 2014.

36. Sun $Y$ and Ma L: The emerging molecular machinery and therapeutic targets of metastasis. Trends Pharmacol Sci 36: 349-359, 2015.

37. Kakarala M and Wicha MS: Implications of the cancer stem-cell hypothesis for breast cancer prevention and therapy. J Clin Oncol 26: 2813-2820, 2008

38. Chen Y, Zhang F, Tsai Y, Yang X, Yang L, Duan S, Wang X, Keng P and Lee SO: IL-6 signaling promotes DNA repair and prevents apoptosis in $\mathrm{CD}_{133^{+}}$stem-like cells of lung cancer after radiation. Radiat Oncol 10: 227, 2015.

39. Roudi R, Korourian A, Shariftabrizi A and Madjd Z: Differential expression of cancer stem cell markers ALDH1 and CD133 in various lung cancer subtypes. Cancer Invest 33: 294-302, 2015.

40. Liu QF, Zhang ZF, Hou GJ, Yang GY and He Y: Polymorphisms of the stem cell marker gene CD133 and the risk of lung cancer in Chinese population. Lung 194: 393-400, 2016.

41. Nian WQ, Chen FL, Ao XJ and Chen ZT: CXCR4 positive cells from Lewis lung carcinoma cell line have cancer metastatic stem cell characteristics. Mol Cell Biochem 355: 241-248, 2011.
42. Jung MJ, Rho JK, Kim YM, Jung JE, Jin YB, Ko YG, Lee JS, Lee SJ, Lee JC and Park MJ: Upregulation of CXCR4 is functionally crucial for maintenance of stemness in drug-resistant non-small cell lung cancer cells. Oncogene 32: 209-221, 2013.

43. Wang Z, Sun J, Feng Y, Tian X, Wang B and Zhou Y: Oncogenic roles and drug target of CXCR4/CXCL12 axis in lung cancer and cancer stem cell. Tumour Biol 37: 8515-8528, 2016.

44. Jiang YX, Yang SW, Li PA, Luo X, Li ZY, Hao YX and Yu PW: The promotion of the transformation of quiescent gastric cancer stem cells by IL-17 and the underlying mechanisms. Oncogene: Aug 15, 2016 (Epub ahead of print). doi: 10.1038/onc.2016.291.

45. Fish KM: Mesenchymal stem cells drive cardiac stem cell chemotaxis, proliferation, and phenotype via CXCR4 and ckit signaling. Circ Res 119: 891-892, 2016.

46. Zhang C, Zhou C, Wu XJ, Yang M, Yang ZH, Xiong HZ, Zhou CP, Lu YX, Li Y and Li XN: Human CD133-positive hematopoietic progenitor cells initiate growth and metastasis of colorectal cancer cells. Carcinogenesis 35: 2771-2777, 2014.

47. Won C, Kim BH, Yi EH, Choi KJ, Kim EK, Jeong JM, Lee JH, Jang JJ, Yoon JH, Jeong WI, et al: Signal transducer and activator of transcription 3-mediated CD133 up-regulation contributes to promotion of hepatocellular carcinoma. Hepatology 62: 1160-1173, 2015.

48. Zhao W, Luo Y, Li B and Zhang T: Tumorigenic lung tumorospheres exhibit stem-like features with significantly increased expression of CD133 and ABCG2. Mol Med Rep 14: 2598-2606, 2016.

49. Yuan X, Wu H, Han N, Xu H, Chu Q, Yu S, Chen Y and Wu K: Notch signaling and EMT in non-small cell lung cancer: Biological significance and therapeutic application. J Hematol Oncol 7: 87, 2014.

50. Knapp CF, Sayegh Z, Schell MJ, Rawal B, Ochoa T, Sondak VK and Messina JL: Expression of CXCR4, E-cadherin, Bcl-2, and survivin in Merkel cell carcinoma: An immunohistochemical study using a tissue microarray. Am J Dermatopathol 34: 592-596, 2012.

51. Havel LS, Kline ER, Salgueiro AM and Marcus AI: Vimentin regulates lung cancer cell adhesion through a VAV2-Rac1 pathway to control focal adhesion kinase activity. Oncogene 34: 1979-1990, 2015 